\title{
ESSENTIAL LOOPS IN TAUT IDEAL TRIANGULATIONS
}

\author{
SAUL SCHLEIMER AND HENRY SEGERMAN
}

\begin{abstract}
In this note we combinatorialise a technique of Novikov. We use this to prove that, in a three-manifold equipped with a taut ideal triangulation, any vertical or normal loop is non-trivial in the fundamental group.
\end{abstract}

\section{INTRODUCTION}

The notion of a taut ideal triangulation of a three-manifold is due to Lackenby [7]. He combinatorialised the angle structures introduced independently by Casson and by Rivin [12]. They in turn linearised the geometric triangulations of Thurston [13]. Each of these structures plays an important role in modern low-dimensional topology. In particular, taut ideal triangulations have a strong connection to the subject of taut foliations, introduced by Gabai [5], and to that of taut branched surfaces, due to Oertel [10]. In addition to the results of Lackenby, taut ideal triangulations play a central role in the theory of layered triangulations. One spectacular contribution has been as a prerequisite for Agol's theory of veering triangulations [1].

Novikov [9, Theorem 6.1] gives one of the early applications of foliations to the study of the fundamental group of a manifold. He starts with a loop $\delta$ in good position with respect to a foliation $\mathcal{F}$. He further supposes that $H: D \rightarrow M$ is a null-homotopy of $\delta$, also in good position. Pulling back, he obtains a singular foliation $H^{-1}(\mathcal{F})$ on the disk $D$. The Poincaré-Hopf theorem gives combinatorial control of the singularities, which translates to topological control over the homotopy. Morally, the positivity of the Euler characteristic of the disk constrains the position of $\delta$. We refer to Candel and Conlon [4, Chapter 9] for a history of the subject and for detailed proofs.

We introduce a combinatorial version of the Novikov technique; instead of pulling back a foliation we pull back a taut ideal triangulation. This gives a train track with stops in the disk $D$. We so obtain a very

Date: January 11, 2022.

This work is in the public domain. 
simple proof of a variant of one of Novikov's results. That is, suppose that $M$ is a three-manifold, equipped with a taut ideal triangulation $\mathcal{T}$. Let $\mathcal{B}=\mathcal{T}^{(2)}$ be the resulting branched surface in $M$.

Theorem 3.2. Any loop $\delta$ in $M$ which is vertical with respect to $\mathcal{B}$ is non-trivial in $\pi_{1}(M)$.

There is also an indirect proof of this using Novikov's original technique [3, Theorem 4.35(3)], once we observe that $\mathcal{B}$ carries an essential lamination which extends to a taut foliation of $M$ (see [6, Example 5.1] as well as [7, page 373]).

Using our techniques we also obtain a new result, as follows.

Theorem 5.1. Any loop $\gamma$ in $M$ which is normal with respect to $\mathcal{B}$ is non-trivial in $\pi_{1}(M)$.

The proof of Theorem 5.1 is more delicate than that of Theorem 3.2; new behaviour near the boundary of $D$ must be dealt with.

From Theorems 3.2 and 5.1 we deduce that vertical, and also normal, loops are infinite order in the fundamental group. Note that this is a bit weaker than the conclusion in the comparable situation of a train track $\tau$ in a surface - there loops dual to, or carried by, $\tau$ are not only non-trivial but also non-peripheral.

We have a simple corollary of Theorem 5.1. Let $\widetilde{M}$ be the universal cover of $M$ and let $\widetilde{\mathcal{B}}$ be the resulting branched surface.

Corollary 1.1. Suppose that $F$ is a connected surface (perhaps with boundary) carried by $\widetilde{\mathcal{B}}$ and realised as a (perhaps finite) union of faces of $\widetilde{\mathcal{B}}$. Then $F$ is a disk.

Previous work. Gabai and Oertel prove that laminations carried by essential branched surfaces are $\pi_{1}$-injective [6, Lemma 2.7]. Our Theorem 5.1 is both more and less general than their work. We do not require a lamination. They do not require the manifold to be cusped.

Calegari [2, Remark 5.6] gives a very different combinatorial version of Theorem 3.2, in the closed case. He introduces the notion of a local orientation; this is, in a sense, dual to having a transverse taut branched surface $\mathcal{B} \subset M$ where all components of $M-\mathcal{B}$ are taut balls.

Acknowledgements. We thank Marc Lackenby for helpful conversations. The second author was supported in part by National Science Foundation grant DMS-1708239.

\section{BACKGROUND}

Throughout the paper we will use $M$ to denote a compact connected manifold with non-empty boundary. All boundary components will be 
tori or Klein bottles. Suppose that $\mathcal{T}$ is a three-dimensional triangulation; that is, a collection of model tetrahedra and a collection of face pairings. We will also call the faces of a model tetrahedron model faces, and similarly for its edges and vertices.

Let $|\mathcal{T}|$ be the quotient space: that is, we take the disjoint union of the model tetrahedra of $\mathcal{T}$ and identify model faces using the face pairings. Let $\mathcal{T}^{(k)}$ be the $k$-skeleton of $|\mathcal{T}|$. Let $n\left(\mathcal{T}^{(0)}\right)$ be an open regular neighbourhood of the vertices of $\mathcal{T}$. We call $\mathcal{T}$ a ideal triangulation of $M$ if $|\mathcal{T}|-n\left(\mathcal{T}^{(0)}\right)$ is homeomorphic to $M$.

A taut angle structure on $\mathcal{T}$ is an assignment of dihedral angles, zero or $\pi$, to each model edge in $\mathcal{T}$. The assignment is required to obey two conditions. The edge equalities state that, for an edge $e \in \mathcal{T}^{(1)}$, the sum of the dihedral angles of its models is $2 \pi$. The triangle equalities state that, for any model vertex, the sum of the dihedral angles of the three adjacent model edges is $\pi$. We say that the tetrahedra of $\mathcal{T}^{(3)}$ are taut. See Figure 2.1a.

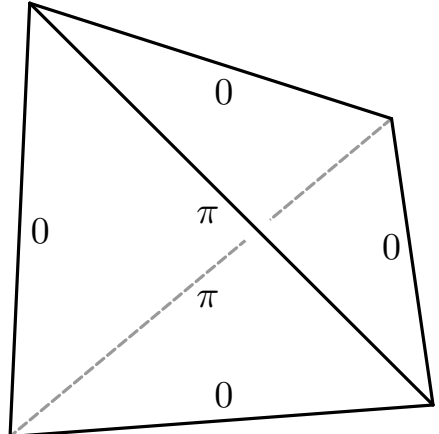

(A) A taut tetrahedron.

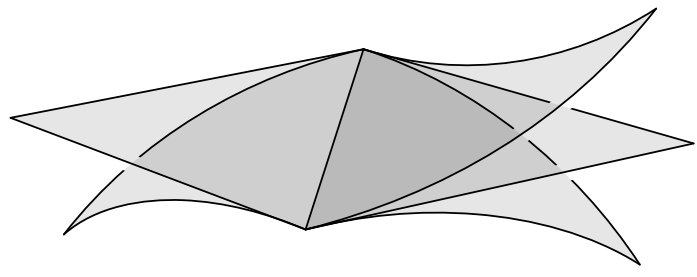

(B) All faces meeting a single edge in $\mathcal{B}$.

FiguRE 2.1

We deduce that every taut tetrahedron has four edges with dihedral angle zero. We call the union of these four edges the equator of the taut tetrahedron.

Suppose now that $e$ is an edge of $\mathcal{T}^{(1)}$. There are exactly two model edges for $e$ with angle $\pi$; all others are zero. Obeying these dihedral angles, we isotope the two-skeleton $\mathcal{T}^{(2)}$ to obtain a smooth branched surface $\mathcal{B}$. See Figure 2.1b. Some references would call $\mathcal{B}$ a non-generic branched surface without vertices. See for example [3, Section 6.3].

Definition 2.2. Suppose that $\delta$ is a smooth embedded loop in $M$. Suppose that $\delta$ is transverse to, and meets, $\mathcal{B}$. Suppose that for every tetrahedron $t$ we have that every arc $d$ of $\delta \cap t$ links the equator of $t$. 
(That is, the endpoints of $d$ are separated in $\partial t$ by the equator of $t$.) Then we say that the loop $\delta$ is vertical with respect to $\mathcal{B}$.

Definition 2.3. Suppose that $\gamma$ is a smooth loop immersed in $\mathcal{B}$. Suppose that $\gamma$ is transverse to, and meets, the edges of $\mathcal{B}$. Suppose that for every model face $f$ of $\mathcal{B}$ and for every component $J$ of $\gamma^{-1}(f)$, the arc $\gamma \mid J$ is normal in $f$. (That is, the endpoints of $\gamma \mid J$ lie in distinct edges of $f$.) Then we say that the loop $\gamma$ is normal with respect to $\mathcal{B}$.

\section{Combinatorics OF NUll-homotopies}

Suppose that $\delta$ is a loop in $M$ which is transverse to the branched surface $\mathcal{B}$. Let $D=D^{2}$ be the unit disk with the usual orientation. Suppose that $H: D \rightarrow M$ is a null-homotopy of $\delta$. We homotope $H$ relative to $\partial D$ to make $H$ transverse to $\mathcal{B}$.

We define $\tau=H^{-1}(\mathcal{B})$. Thus $\tau$ is a train track in $D$. The switches of $\tau$ are exactly the points of $H^{-1}\left(\mathcal{B}^{(1)}\right)$. The stops of $\tau$ are exactly the points of $(H \mid \partial D)^{-1}(\mathcal{B})$. The standard reference for train tracks is [11]; we also rely on [8]. We note that our track $\tau$ does not satisfy the so-called "geometry-condition" [11, page 5], [8, page 52].

We call a connected component $R$ of $D-\tau$ a region. Let $\operatorname{cusps}(R)$ and corners $(R)$ count the number of (necessarily outwards) cusps and corners on the boundary of $R$. As a bit of terminology, we divide $\partial R$ into sides: these are the components of $\partial R$ minus all outward cusps and corners. Note that a side $s$ of $R$ may be a union of several branches of $\tau$.

We define the index of $R$ to be

$$
\operatorname{ind}(R)=\chi(R)-\frac{\operatorname{cusps}(R)}{2}-\frac{\operatorname{corners}(R)}{4}
$$

In Table 1 we give pictures of, and names to, all possible disk regions with non-negative index. Note that index is additive under taking the union of regions [8, page 57]. Thus the sum of the indices of the regions of $D-\tau$ is exactly $\chi(D)$; that is, one. We deduce from this that there is at least one region $R$ with positive index.

Let $r(H)$ be the number of regions of $D-\tau$. Over all null-homotopies of $\delta$, transverse to $\mathcal{B}$, we choose $H$ to minimise $r(H)$. We call such an $H$ minimal.

Lemma 3.1. Suppose that $\delta$ is a loop in $M$ transverse to $\mathcal{B}$. Suppose that $H: D \rightarrow M$ is a minimal null-homotopy of $\delta$. Let $\tau=H^{-1}(\mathcal{B})$. Suppose that $R$ is a region of $D-\tau$. Then we have the following.

(1) $R$ is a disk. 


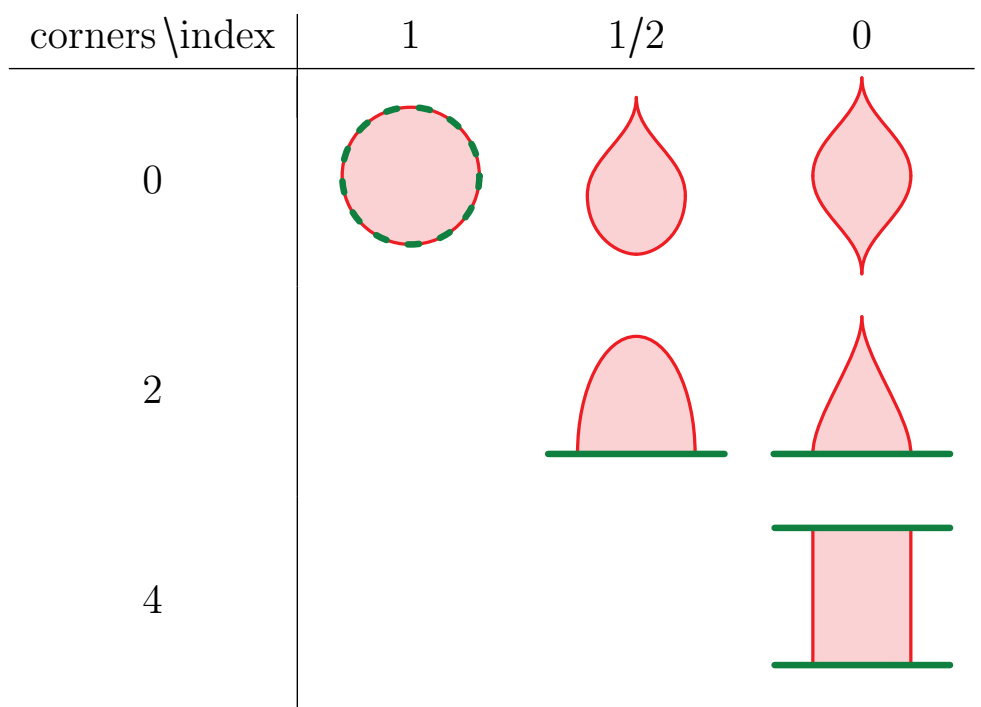

TABLE 1. Disk regions with non-negative index, organised by the number of their corners. These are named as follows: nullgon, cusped monogon, cusped bigon, boundary bigon, boundary trigon, and rectangle. If $\tau$ is empty then $D$ is called a complete nullgon.

(2) Suppose that $s$ is a side of $R$. Then the interior of $s$ meets at most one switch.

(3) If $R$ is a nullgon, then $R=D$ is a complete nullgon.

(4) $R$ is not a cusped monogon.

Thus, the positive index regions of $D$ are either a single complete nullgon, or a non-empty collection of boundary bigons.

Proof.

(1) If $R$ has topology, then we can compress it into the containing tetrahedron and reduce $r(H)$.

(2) Suppose that the interior of $s$ meets at least two switches. All such switches in the interior of $s$ are preimages under $H$ of a single edge. Hence there is a branch $b \subset \tau$ so that $H(b)$ is a non-normal arc. We homotope $H$ in a neighbourhood of $b$ to make $H(b)$ simple. This done, $H(b)$ cuts a bigon $B$ off of the face containing $H(b)$. We then homotope $H$ across $B$. This does not increase $r(H)$. If $r(H)$ does not decrease, then this move disconnects $\tau$, and creates a region with topology, contradicting (1). 
(3) Suppose that $R$ is a nullgon, not equal to $D$. If $H(\partial R)$ is disjoint from $\mathcal{B}^{(1)}$ then the region adjacent to $R$ is not a disk, contradicting (1). It follows that $\partial R$ consists of an even number of branches of $\tau$ (alternating between the two faces of a tetrahedron $t$ on either side of a $\pi$-edge of $t$ ). But this contradicts (2).

(4) Suppose that $t$ is the taut tetrahedron containing $H(R)$. Let $s$ be the boundary of $R$. We deduce that the loop $s$ crosses the equator of $t$ exactly once, a contradiction.

Thus, the only possible positive index regions are complete nullgons and boundary bigons.

Equipped with this we can now prove the following.

Theorem 3.2. Let $(M, \mathcal{T})$ be a three-manifold equipped with a taut ideal triangulation. Let $\mathcal{B}=\mathcal{T}^{(2)}$ be the resulting branched surface in $M$. Any loop $\delta$ in $M$ which is vertical with respect to $\mathcal{B}$ is non-trivial in $\pi_{1}(M)$.

Proof. Suppose that $H: D \rightarrow M$ is a minimal null-homotopy of the vertical loop $\delta$. Applying Lemma 3.1, there must be a region $R$ of $D-\tau$ which is a boundary bigon. Let $t$ be the tetrahedron containing $H(R)$. Let $d=\partial R \cap \partial D$ and let $s=\partial R-d^{\circ}$. From the definition of vertical, we have that $H(d)$ links the equator of $t$. Therefore $H(s)$ crosses the equator of $t$ an odd number of times, and thus at least once. This contradicts the fact that $\partial R$ has no cusps.

\section{TRAnsverse taut}

In order to prove Theorem 5.1, we will use the following strengthening of the notion of a taut structure. A transverse taut structure on $\mathcal{T}$ is a taut structure together with a co-orientation on $\mathcal{B}$ with the following property. If model faces $f$ and $f^{\prime}$ of a model tetrahedron $t$ share a common model edge $e$, then

- the edge $e$ is part of the equator of $t$ if and only if exactly one of the co-orientations on $f$ and $f^{\prime}$ points into $t$.

See Figure 4.1a. It follows that the co-orientations on faces incident to an edge change direction precisely twice as we go around an edge. See Figure 4.1b.

Suppose that $\mathcal{T}$ is an ideal triangulation of a manifold $M$ equipped with a taut structure. We now construct a triangulation $\widetilde{\mathcal{T}}$ of a double cover $\widetilde{M}$ of $M$. By construction, the lift of the taut structure on $\mathcal{T}$ to $\widetilde{\mathcal{T}}$ will support a transverse taut structure.

For each taut tetrahedron $t$ of $\mathcal{T}$, we arbitrarily label the two model edges with dihedral angle $\pi$ as $e^{\prime}$ and $e^{\prime \prime}$. In $\widetilde{\mathcal{T}}$, we have two taut 


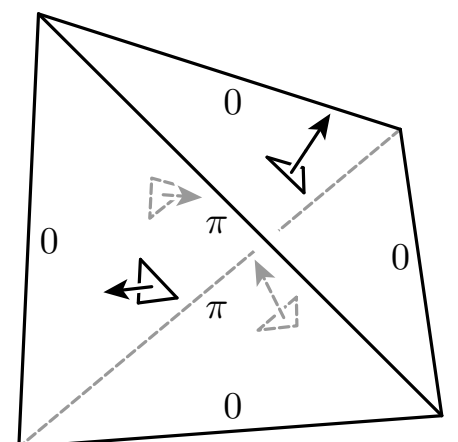

(A) Co-orientations and angles in a transverse taut tetrahedron.

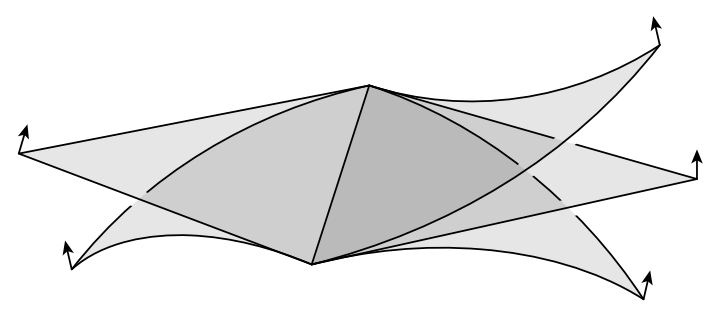

(B) Co-orientations around an edge.

FIGURE 4.1

tetrahedra $t^{\prime}$ and $t^{\prime \prime}$ corresponding to $t$. We assign a co-orientation to the model faces of $t^{\prime}$ and $t^{\prime \prime}$ in such a way that the co-orientation points into the tetrahedron on the two model faces of $t^{*}$ incident to $e^{*}$. Now suppose that $t_{i}$ and $t_{j}$ are tetrahedra of $\mathcal{T}$, glued to each other along model faces $f_{i}$ and $f_{j}$. In $\widetilde{\mathcal{T}}$ we have tetrahedra $t_{i}^{\prime}, t_{i}^{\prime \prime}, t_{j}^{\prime}$, and $t_{j}^{\prime \prime}$, with model faces $f_{i}^{\prime}, f_{i}^{\prime \prime}, f_{j}^{\prime}$, and $f_{j}^{\prime \prime}$, respectively.

We glue $t_{i}^{\prime}$ to either $t_{j}^{\prime}$ or $t_{j}^{\prime \prime}$ as the co-orientation on $f_{i}^{\prime}$ agrees with $f_{j}^{\prime}$ or $f_{j}^{\prime \prime}$. We similarly glue $t_{i}^{\prime \prime}$ to the remaining copy of $t_{j}$. Having made all such gluings, the resulting triangulation $\widetilde{\mathcal{T}}$ has a transverse taut structure by construction. It has one component if and only if the taut structure on $\mathcal{T}$ does not support a transverse taut structure.

\section{Proof of The MAIN RESUlT}

Theorem 5.1. Let $(M, \mathcal{T})$ be a three-manifold equipped with a taut ideal triangulation. Let $\mathcal{B}=\mathcal{T}^{(2)}$ be the resulting branched surface in $M$. Any loop $\gamma$ in $M$ which is normal with respect to $\mathcal{B}$ is non-trivial in $\pi_{1}(M)$.

Proof. Suppose for a contradiction, that the normal loop $\gamma$ is nullhomotopic. Thus $\gamma$ lifts to a normal loop in any cover. Thus, without loss of generality, we may assume that the taut structure on $\mathcal{T}$ supports a transverse taut structure. This gives us a local notion of upwards. In particular, every model tetrahedron has two lower faces and two upper faces, separated by its equator.

Lemma 3.1 does not apply directly to a normal loop $\gamma$. So, let $A$ be a model annulus with horizontal boundary circles $\partial_{0} A \sqcup \partial_{1} A$. Let $G$ be a 
small smooth homotopy $G: A \rightarrow M$, moving $\gamma$ slightly upwards. That is, $G\left(\partial_{0} A\right)=\gamma$ and we define $\delta=G\left(\partial_{1} A\right)$. We ensure that $G$ is transverse to $\mathcal{B}$ away from $\partial_{0} A$; also, we arrange that for each vertical interval $J$ in $A$ the tangents to $G(J)$ point upwards. We will apply Lemma 3.1 to $\delta$.

We call $\delta$ a raised curve. We call the components of $\delta-\mathcal{B}$ raised arcs. There are six types of raised arc. These are shown in Figure 5.2. There is a cellulation of $A$ with one-skeleton $\partial A \cup G^{-1}(\mathcal{B})$. Suppose that $C$ is a two-cell. Let $c=C \cap \partial_{0} A$ and $d=C \cap \partial_{1} A$. Thus $G(c) \subset \gamma$ and $G(d) \subset \delta$. We say that $G(c)$ is the lowering of the raised arc $G(d)$. We record this by the lowering map, $L$, where $L(G(d))=G(c)$. Note that $G(c)$ may be either a single vertex, a single normal arc, or two normal arcs. Again, see Figure 5.2.

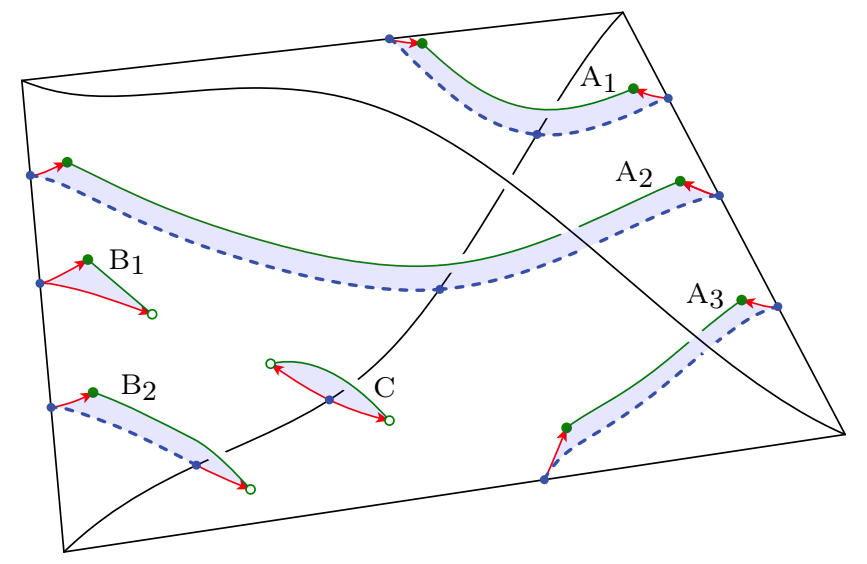

Figure 5.2. A taut tetrahedron containing the six possible types of raised $\operatorname{arcs}$ of $\delta$. These are drawn in (solid) green. The normal arcs (or points) of $\gamma$, namely the lowerings of the raised arcs, are drawn in (dashed) blue. Images of the two-cells of the annulus $A$ are shaded in light blue. Filled green dots indicate endpoints of raised arcs on the top two faces of the tetrahedron; open green dots indicate endpoints on the bottom two faces.

Suppose that $H: D \rightarrow M$ is a minimal null-homotopy of $\delta$. Recall that $\tau=H^{-1}(\mathcal{B})$. Applying Lemma 3.1, we find that $D-\tau$ has at least two boundary bigons. Applying another small homotopy, we can retain minimality and also make $H$ transverse to $\gamma$.

Pulling back the transverse taut structure on $\mathcal{B}$ by $H$ gives a transverse orientation on the branches of $\tau$ which is consistent across switches. Thus, for any region $R$ of $D-\tau$ and for any side $s$ of $R$, the transverse orientation on $s$ points either into, or out of, $R$. This gives us a classification of boundary bigons. Suppose that $R$ is a boundary bigon and $s=\partial R-\partial D$ is its side in $\tau$. If the transverse orientation on $s$ points 
out of $R$ then we call $R$ a min-bigon. If it points into $R$ we call $R$ a max-bigon.

5.3. Min-bigons. Suppose that $R$ is a min-bigon. We move $\gamma$ up, across $H(R)$, to obtain $\gamma^{\prime}$. We appeal to Lemma 3.1(2) to ensure that $\gamma^{\prime}$ is normal. Let $\delta^{\prime}$ be the corresponding raised loop and let $H^{\prime}$ be the new null homotopy. See Figure 5.4.

The loop $\gamma^{\prime}$ may be shorter than, the same length as, or longer than $\gamma$ (see types $\mathrm{A}_{1}, \mathrm{~A}_{2}$ and $\mathrm{A}_{3}$ in Figure 5.2). However, $H^{\prime}$ has exactly one fewer region. That is, $r\left(H^{\prime}\right)=r(H)-1$. We repeat this process until there are no more min-bigons.
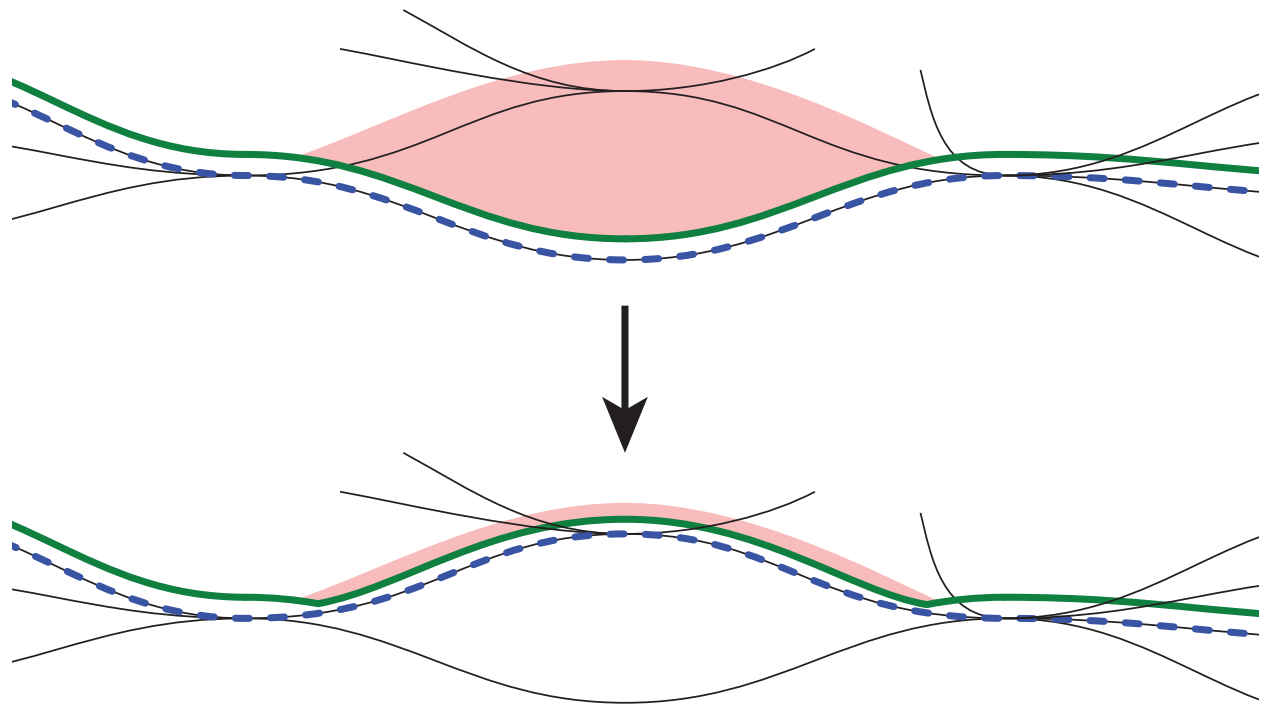

Figure 5.4. Pushing over a min-bigon of type $A_{3}$.

5.5. Max-bigons. Suppose that $R_{0}$ is a max-bigon. Unlike the situation of a min-bigon, a max-bigon does not give us a simple move to reduce complexity. The asymmetry stems from the fact that we raised $\gamma$ rather than lowered it. Instead, our plan is to uniquely associate to $R_{0}$ two small subregions of $D-\tau$, each with index $-1 / 4$. This will imply that the index of $D$ is at most zero. This contradiction finally proves Theorem 5.1.

We begin as follows. Let $s$ be the side of $R_{0}$ in $\tau$. Let $d_{0}=\partial R_{0}-s \subset \partial D$. We give $d_{0}$ the (tangential) orientation it receives from $D$. In Figure 5.7, this orientation will point left. Note that $H\left(d_{0}\right) \subset \delta$ is a raised arc. Let $c_{0}=L\left(H\left(d_{0}\right)\right)$ be its lowering. 
Claim 5.6.

- The raised arc $H\left(d_{0}\right)$ has type C.

- The side $s$ meets exactly one switch $c_{0}^{\prime}$ of $\tau$.

- The vertices $c_{0}$ and $H\left(c_{0}^{\prime}\right)$ cobound a sub-edge $\epsilon_{0} \subset \mathcal{B}^{(1)}$.

Proof. Let $t_{0}$ be the tetrahedron containing $H\left(R_{0}\right)$. By the definition of a max-bigon the transverse orientation on $s$ points into $R_{0}$. Thus each corner of $H\left(R_{0}\right)$ is contained in a lower face of $t_{0}$. Consulting Figure 5.2 we deduce that $H\left(d_{0}\right)$ is of type C. Thus each corner of $H\left(R_{0}\right)$ is contained in its own lower face of $t_{0}$. We deduce that $s$ meets at least one switch of $\tau$. By Lemma 3.1(2) the side $s$ meets exactly one switch, which we call $c_{0}^{\prime}$.

Since $H$ is transverse to $\gamma$, the vertices $c_{0}$ and $H\left(c_{0}^{\prime}\right)$ are distinct. They are contained in the same edge of $\mathcal{B}^{(1)}$ : namely the bottom edge $e_{0}$ of $t_{0}$. In $e_{0}$ they cobound a sub-edge, which we call $\epsilon_{0}$.

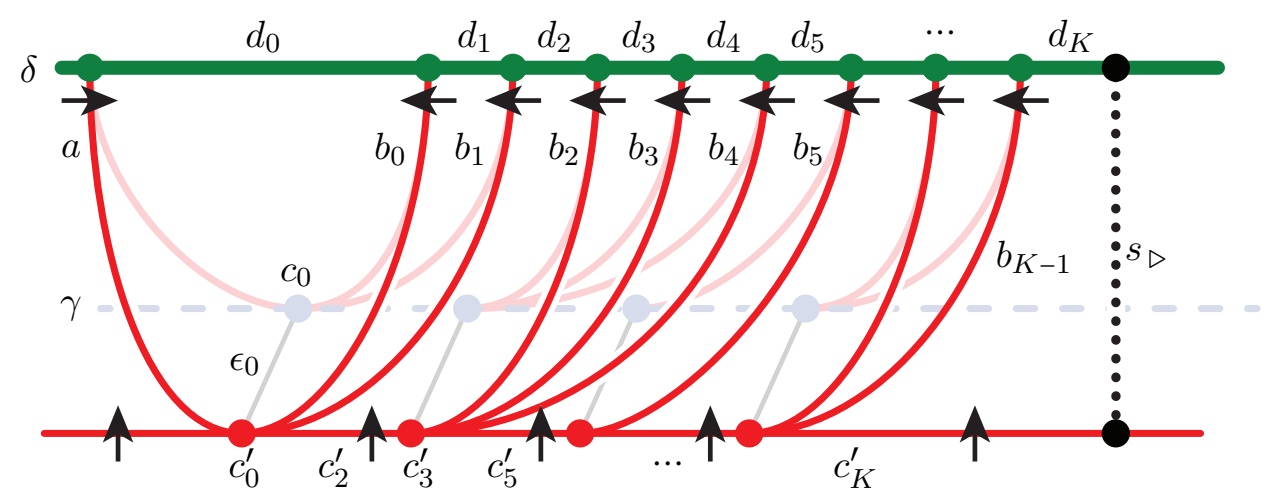

Figure 5.7. A possible picture of part of the annulus $A$ (in back), the bigons $B_{k}$ (in front), and the homotopies $F_{k}$ (bottom). To lighten the notation in this figure, we have omitted applying $H$ to labels of subsets of $D$. Transverse orientations on the branches $b_{i}$ are shown with arrows. Note that $\epsilon_{0}=\epsilon_{1}$, $c_{0}^{\prime}=c_{1}^{\prime}, c_{3}^{\prime}=c_{4}^{\prime}$, and so on.

Let $a$ and $b_{0}$ be the components of $s-c_{0}^{\prime}$, where $b_{0}$ meets the right endpoint of $d_{0}$. See the leftmost region on Figure 5.7.

Now consider a sequence of regions $R_{0}, R_{1}, \ldots, R_{n}$ that meet $\partial D$ in the sides $d_{0}, d_{1}, \ldots d_{n}$ as we move along $\partial D$ to the right. Define $c_{k}=L\left(H\left(d_{k}\right)\right) \subset \gamma$ : the lowering of the raised arc $H\left(d_{k}\right)$. Define $\gamma_{k}=\cup_{i=0}^{k} c_{i} \subset \gamma$.

Let $b_{i}$ be the branch of $\tau$ that meets $\partial D$ at the right corner of $R_{i}$. We now choose $n=N$ such that $b_{i-1}$ and $b_{i}$ have the same transverse orientation for $1 \leq i<N$, while they have opposite transverse orientations for $i=N$. Thus, $R_{N}$ is at a local minimum of $\gamma$, and we are going downhill to it from the local maximum at $R_{0}$. This downhill condition 
implies that for $i \in[1, N)$, the raised arc $H\left(d_{i}\right)$ is of type either $\mathrm{B}_{1}$ or $\mathrm{B}_{2}$. Again, see Figure 5.2.

Recall that all positive index regions are now max-bigons. Thus none of the $R_{i}$ can have positive index for $i>0$. Let $K$ be the smallest number for which $R_{K}$ has negative index, or if there is none, then set $K=N$.

Claim 5.8. The region $R_{K}$ is not a boundary trigon.

Proof. If $K<N$ then by definition, $R_{K}$ has negative index, and so is not a boundary trigon. If $K=N$ then $R_{N}$ cannot be a boundary trigon since the transverse orientations on the two sides of a boundary trigon must agree, yet $R_{N}$ is at a local minimum of $\gamma$.

For all $k \in[0, K)$ we define the union $B_{k}=\cup_{i=0}^{k} R_{i}$. Define $\gamma_{k}^{\prime}=$ $\partial B_{k}-\left(a^{\circ} \cup b_{k}^{\circ} \cup \partial D\right)$. (Unlike in Section 5.3, here $\gamma_{k}^{\prime}$ is a push-off of only a section of $\gamma$.)

Definition 5.9. Suppose that $g, h:[0,1] \rightarrow \mathcal{B}$ are paths. Suppose that $F:[0,1] \times[0,1] \rightarrow \mathcal{B}$ is a homotopy from $g$ to $h$. Thus $g(x)=F(x, 0)$ and $h(x)=F(x, 1)$. We say that $F$ is transverse if whenever $F\left(x_{0}, t_{0}\right)$ is contained in a (1- or 2-) cell $C$ of $\mathcal{B}$, we have that the trace $F\left(x_{0},[0,1]\right)$ lies in $C$.

Claim 5.10. For all $k \in[1, K)$ :

(1) The region $R_{k}$ is a boundary trigon.

(2) The union $B_{k}$ has exactly two corners and no cusps.

(3) There is a transverse homotopy $F_{k}$ taking $\gamma_{k}$ to $H\left(\gamma_{k}^{\prime}\right)$.

Proof. We will prove this by induction. Claim 5.6 implies the base case (for $k=1$ ) in a manner essentially identical to the general inductive step, so we omit its proof.

Suppose that the hypotheses hold at step $k$. Recall that $H\left(d_{k}\right)$ has type $\mathrm{B}_{1}$ or $\mathrm{B}_{2}$, so it has precisely one lower endpoint. Let $f_{k}$ be the face that contains the lower endpoint. Let $p$ be the endpoint of $\gamma_{k}$, and let $e_{k}$ be the edge of $f_{k}$ containing $p$. Let $\beta$ be the normal arc of $\gamma$ immediately after $p$. Let $f_{\beta}$ be the face containing $\beta$. Viewed in a small neighbourhood of $e_{k}$, the faces $f_{\beta}$ and $f_{k}$ are on the same side (say the right side) of $e_{k}$, and $f_{\beta}$ is below $f_{k}$.

Let $p^{\prime}$ be the endpoint of $\gamma_{k}^{\prime}$ meeting $b_{k}$. By hypothesis (3), the transverse homotopy $F_{k}$ takes $p$ to $H\left(p^{\prime}\right)$, with trace lying in $e_{k}$. Since $H$ is transverse to $e_{k}$ at $H\left(p^{\prime}\right)$, we deduce that $H(D)$ meets both $f_{k}$ and $f_{\beta}$ at $H\left(p^{\prime}\right)$. Thus $p^{\prime}$ is a switch of $\tau$ with a cusp immediately below $b_{k}$, to the right of $p^{\prime}$, pointing at $\gamma_{k}^{\prime}$ (which extends to the left 
of $p^{\prime}$ ). This cusp lies in $R_{k+1}$, since $b_{k}$ is part of the boundary of $R_{k+1}$. See Figure 5.7.

If $R_{k+1}$ has negative index then $k+1=K$ and we have nothing to prove. So suppose that $R_{k+1}$ has index zero. Consulting Table 1 we deduce that $R_{k+1}$ is a boundary trigon. This proves hypothesis (1). Note that hypothesis (2) follows because $B_{k}$ meets $R_{k+1}$ along $b_{k}$.

Let $s_{k+1}=\partial R_{k+1}-\left(d_{k+1} \cup b_{k}^{\circ}\right)$ be the remaining side of the boundary trigon $R_{k+1}$. By Lemma 3.1(2) there is at most one switch in the interior of $s_{k+1}$. Let $c_{k+1}^{\prime}=s_{k+1}-b_{k+1}^{\circ}-\partial D$. Note that $\gamma_{k+1}^{\prime}=\gamma_{k}^{\prime} \cup c_{k+1}^{\prime}$.

The path $H\left(s_{k+1}\right)$ has endpoints $H\left(p^{\prime}\right)$ and the lower endpoint of $H\left(d_{k+1}\right)$. The point $H\left(p^{\prime}\right)$ lies on the edge $e_{k}$. Recall that $f_{k+1}$ is the face containing the lower endpoint of $H\left(d_{k+1}\right)$. There are two cases, depending on the type of $H\left(d_{k+1}\right)$.

- Suppose that $H\left(d_{k+1}\right)$ has type $\mathrm{B}_{1}$. Then $\gamma_{k+1}=\gamma_{k}$. In this case, $e_{k}$ is a boundary edge of $f_{k+1}$. Since there is at most one switch in the interior of $s_{k+1}$, there are in fact no such switches. So $s_{k+1}=b_{k+1}$ and $c_{k+1}^{\prime}$ is a single switch, equal to $p^{\prime}$. We deduce that $\gamma_{k+1}^{\prime}=\gamma_{k}^{\prime}$. Since $\gamma_{k+1}=\gamma_{k}$ and $\gamma_{k+1}^{\prime}=\gamma_{k}^{\prime}$, we set $F_{k+1}=F_{k}$. See Figure 5.11a.

- Suppose that $H\left(d_{k+1}\right)$ has type $\mathrm{B}_{2}$. Then $\gamma_{k+1}=\gamma_{k} \cup c_{k+1}$. Let $t_{k+1}$ be the tetrahedron containing $H\left(R_{k+1}\right)$. In this case, the path $H\left(s_{k+1}\right)$ must cross the bottom edge of $t_{k+1}$ in order to get into $f_{k+1}$. Since there is at most one switch in the interior of $s_{k+1}$, there is exactly one. Let $f$ be the other lower face of $t_{k+1}$. Thus $c_{k+1}$ is a normal arc in $f$. Note that $H\left(c_{k+1}^{\prime}\right)$ is a properly immersed arc in $f$, with endpoints on the same edges as those of $c_{k+1}$. Thus there is a transverse homotopy $E$ taking $c_{k+1}$ to $H\left(c_{k+1}^{\prime}\right)$. Reparametrising $E$, we set $F_{k+1}=F_{k} \cup E$. See Figure 5.11b.

This proves hypothesis (3).

Let $B_{\triangleright}=B_{K-1}$. This is the right-bigon for $R_{0}$. We rerun the argument of Claim 5.10 to the left to obtain the left-bigon for $R_{0}$, denoted by $B_{\triangleleft}$.

The induction in the proof of Claim 5.10 extends to show that $R_{K}$ contains a cusp pointing at $\gamma_{K-1}^{\prime}$. The cusp lies between $b_{K-1}$ and another branch on the boundary of $R_{K}$, which we call $c_{K}^{\prime}$. See the far right of Figure 5.7. Let $Q_{\triangleright}$ be a small closed regular neighbourhood of $b_{K-1}$ in $R_{K}$. The boundary of the subregion $Q_{\triangleright}$ has four sides; we call it a right-quadrilateral. The four sides are $d_{K} \cap N, b_{K-1}, c_{K} \cap N$, and a fourth side, $s_{\triangleright}$ say. Note that $s_{\triangleright}$ is properly embedded in $R_{K}$. The quadrilateral $Q_{\triangleright}$ therefore has one cusp and three corners, and so it has index $-1 / 4$. An identical argument builds the left-quadrilateral $Q_{\triangleleft}$. 


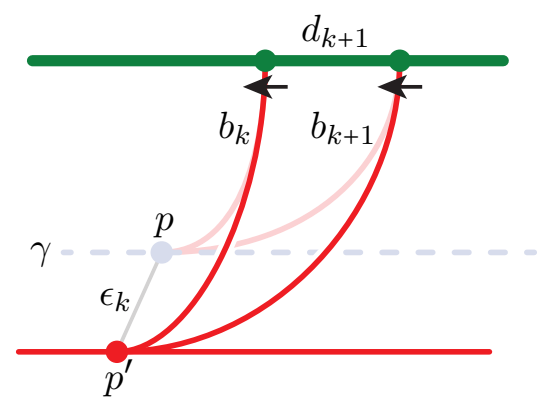

(A) $H\left(d_{k+1}\right)$ has type $\mathrm{B}_{1}$.

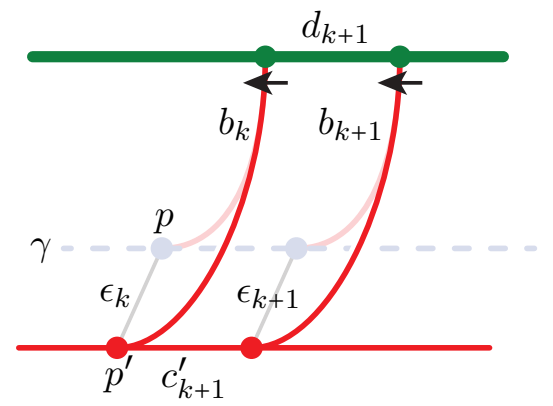

(B) $H\left(d_{k+1}\right)$ has type $\mathrm{B}_{2}$.

Figure 5.11. Extending the transverse homotopy $F_{k}$. As in Figure 5.7, we have omitted applying $H$ to labels of subsets of $D$.

Let $S\left(R_{0}\right)=Q_{\triangleleft} \cup B_{\triangleleft} \cup B_{\triangleright} \cup Q_{\triangleright}$.

Claim 5.12. For any max-bigons $R$ and $R^{\prime}$

(1) $S(R)$ is embedded in $D$,

(2) if $R \neq R^{\prime}$ then $S(R)$ and $S\left(R^{\prime}\right)$ are disjoint, and

(3) $S(R)$ is a rectangle.

Proof. Let $B_{\triangleleft}$ and $B_{\triangleright}$ be the right- and left-bigons for $R$; define $B_{\triangleleft}^{\prime}$ and $B_{\triangleright}^{\prime}$ similarly for $R^{\prime}$. Note that boundary trigons in $B_{\triangleleft}$ have transverse orientations on their branches that disagree with the tangential orientation on $\partial D$. On the other hand, boundary trigons in $B_{\triangleright}^{\prime}$ have transverse orientations that agree with the tangential orientation.

This proves that $B_{\triangleleft}$ and $B_{\triangleright}$ share only one region: the max-bigon itself, and so $B=B_{\triangleleft} \cup B_{\triangleright}$ is again a boundary bigon. The same argument shows that $B$ and $B^{\prime}=B_{\triangleleft}^{\prime} \cup B_{\triangleright}^{\prime}$ have no regions in common if $R \neq R^{\prime}$.

We claim that $\partial B$ and $\partial B^{\prime}$ are disjoint. To see this, note that $\partial B$ consists of an arc in $\partial D$, and an arc in $\tau$. The transverse orientation on the arc in $\tau$ points into $B$, and similarly for $B^{\prime}$.

Let $Q_{\triangleleft}, Q_{\triangleright}, Q_{\triangleleft}^{\prime}$ and $Q_{\triangleright}^{\prime}$ be the quadrilaterals for $R$ and $R^{\prime}$. Since these are obtained by taking subsets of small regular neighbourhoods of branches in $\partial B$ and $\partial B^{\prime}$, these are all pairwise disjoint (if $R \neq R^{\prime}$ ). This proves parts (1) and (2).

Adding the subregions $Q_{\triangleleft}$ and $Q_{\triangleright}$ replaces the two corners of $B$ with four corners, and thus $S(R)$ is a rectangle, and we obtain (3).

Let $D^{\prime}=D-\cup S(R)$, where the union ranges over all max-bigons $R$. Claim 5.13. The induced cellulation of $D^{\prime}$ has no regions of positive index. 
Proof. Suppose that $R^{\prime}$ is a region of $D^{\prime}$ having positive index. If $R^{\prime}$ is a non-complete nullgon, or monogon, then $R^{\prime}$ is also a region of $D-\tau$, contradicting Lemma 3.1. Also, $R^{\prime}$ is not a boundary bigon since we removed them all. Thus $R^{\prime}$ was created by cutting quadrilaterals out of some region $R$ of $D-\tau$. Note that $R^{\prime}$ meets $\tau$, meets $\partial D$ and meets $\partial Q_{\triangleright}$ (say) along some side $s_{\triangleright}$. So $R^{\prime}$ has at least three corners. Since its index is positive, $R^{\prime}$ has exactly three corners. Thus $R=R^{\prime} \cup Q_{\triangleright}$ is a boundary trigon, contradicting Claim 5.8.

Note that $D^{\prime}$ has both outward and inward corners (a combinatorial version of the exterior angle being $3 \pi / 2$ ). Again following [8, page 57], we generalise our definition of index; each inward corner adds $+1 / 4$ to the overall index. Thus $D^{\prime}$ has non-positive index. Since rectangles have index zero, from the additivity of index we deduce that $D$ has nonpositive index, a contradiction. This concludes the proof of Theorem 5.1.

\section{REFERENCES}

[1] Ian Agol. Ideal triangulations of pseudo-Anosov mapping tori. In Topology and geometry in dimension three, volume 560 of Contemp. Math., pages 1-17. Amer. Math. Soc., Providence, RI, 2011. arXiv:1008.1606, doi:10.1090/conm/560/11087. [1]

[2] Danny Calegari. Foliations transverse to triangulations of 3-manifolds. Comm. Anal. Geom., 8(1):133-158, 2000. arXiv:math/9803109, doi:10.4310/CAG.2000.v8. n1.a5. [2]

[3] Danny Calegari. Foliations and the geometry of 3-manifolds. Oxford Mathematical Monographs. Oxford University Press, Oxford, 2007. http://math.uchicago. edu/ dannyc/books/foliations/foliations.html. [2,3]

[4] Alberto Candel and Lawrence Conlon. Foliations. II, volume 60 of Graduate Studies in Mathematics. American Mathematical Society, Providence, RI, 2003. doi : $10.1090 / \mathrm{gsm} / 060$. [1]

[5] David Gabai. Foliations and the topology of 3-manifolds. J. Differential Geom., 18(3):445-503, 1983. doi:10.4310/jdg/1214437784. [1]

[6] David Gabai and Ulrich Oertel. Essential laminations in 3-manifolds. Ann. of Math. (2), 130(1):41-73, 1989. doi:10.2307/1971476. [2]

[7] Marc Lackenby. Taut ideal triangulations of 3-manifolds. Geom. Topol., 4:369395, 2000. arXiv:math/0003132, doi:10.2140/gt.2000.4.369. [1,2]

[8] Lee Mosher. Train track expansions of measured foliations. Preprint, 2003. https: //web.archive.org/web/20190829013413/http://andromeda. rutgers.edu/ mosher/. [4, $14]$

[9] S. P. Novikov. The topology of foliations. Trudy Moskov. Mat. Ob̌̌č., 14:248-278, 1965. [1]

[10] Ulrich Oertel. Homology branched surfaces: Thurston's norm on $H_{2}\left(M^{3}\right)$. In Low-dimensional topology and Kleinian groups (Coventry/Durham, 1984), volume 112 of London Math. Soc. Lecture Note Ser., pages 253-272. Cambridge Univ. Press, Cambridge, 1986. [1] 
[11] R. C. Penner and J. L. Harer. Combinatorics of train tracks, volume 125 of Annals of Mathematics Studies. Princeton University Press, Princeton, NJ, 1992. doi:10.1515/9781400882458. [4]

[12] Igor Rivin. Euclidean structures on simplicial surfaces and hyperbolic volume. Ann. of Math. (2), 139(3):553-580, 1994. doi:10.2307/2118572. [1]

[13] William Thurston. Geometry and topology of three-manifolds. Lecture notes, 1978. http://msri.org/publications/books/gt3m/. [1]

Mathematics Institute, University of Warwick, Coventry CV4 7AL, UNITED KINGDOM

Email address: s.schleimer@warwick.ac.uk

Department of Mathematics, Oklahoma State University, Stillwater, OK, 74078, USA

Email address: segerman@math.okstate.edu 